\title{
Use of radiography images and gray level co- occurrence matrix to investigate gravitational granular flow
}

\author{
Selam Waktola ${ }^{1, *}$, Krzysztof Grudzień ${ }^{1}$, Maciej Niedostatkiewicz ${ }^{2}$, and Laurent Babout $^{1}$ \\ ${ }^{1}$ Lodz University of Technology, Institute of Applied Computer Science, ul. Żeromskiego 116, \\ 90-924 Lodz, Poland \\ ${ }^{2}$ Gdańsk University of Technology, Faculty of Civil and Environmental Engineering, \\ ul. Narutowcza 11/12, 80-233 Gdansk, Poland
}

\begin{abstract}
The paper presents analysis of granular gravitational flow based on radiography images processing. The investigations were conducted for silo model geometry with concentric/eccentric discharging modes. The continuous X-ray radiography scans of granular material distribution, acquired during flow, were obtained by means of an especially designed model silo with rectangular bin and different settings of hopper angles. Image processing involved texture analysis methods, the Gray-Level CoOccurrence Matrix (GLCM). The texture analysis of radiography images provides information about changes of granular porosity in different silo zones during silo discharging process. This technique allows to divide the silo space into a number homogenous regions with similar porosity level. The proposed methodology was applied to analyse the flow in silo model with various hopper angles.
\end{abstract}

\section{Introduction}

A high level of automation of technological processes forces the necessity of storing and collecting materials for their further processing. Most of solid state industrial products appear, at various stages of production, in form of powders or grains [1]. Approximately $60 \%$ of industrial solid materials are stored, transported, or processed as bulk solids [2, 3]. Bulk solid materials are stored in containers; the largest groups are silos and bunkers, very often with reinforced concrete walls. Proper conveying and handling of bulk solids acts significantly on industry and society, where accurate designed silos and process flow control is crucial to efficiency and safety of industrial process and natural environment as well [4-6]. The control of granular flow phenomena in industrial installations has been investigated for many years, but it still remains a challenging task both in practical and theoretical point of view [7,8]. Granular materials are ubiquitous by nature and for a scientific point of view, the study of their flow is seen among key chemical-physical phenomena without answers to many research questions, because of the peculiar interaction between particles during movement [1-3]. The analysis of bulk solid structure behaviour

* Corresponding author: kgrudzi@iiis.p.lodz.pl 
during flow allows to better understand the phenomena occurring in industrial processes. The silo discharging process is frequent in various branches of industry to introduce material in form of powders or grain to production line, after their storing in container. The main tasks of these containers are protection and storing solid materials and also as an important link between transporting lines in many industrial processes $[1,3,5]$. Silos are often used to heating or cooling, aeration or fumigation of powders. In industrial plants silos are applied for product mixing and blending.

Granular material behaviour is influenced by internal and external factors, such as granular properties, container structure, temperate or humidity, initial solid density, wall roughness, mean grain diameter of solid, pressure level, and flow velocity. There is a number of problems with silo utilization from uneven flow to blockage to silo collapse. These eventual disasters caused by uneven flow thus leads to major environmental and economic impacts due to the waste created [1]. This may be caused by the un- even material distribution, resulting in unsymmetrical stresses of walls and the bottom during loading and discharging of bulk materials. The main reasons influencing on stability of the silo is non-symmetrical flow of the bulk solid and non-controlled volume changes in the hopper $[9,10]$. Both of them are connected with the shape and the dimension of the core of flow, created from the beginning of silo emptying process. The knowledge about the flow profile and mechanism of concentration changes, also at the core of flow allows to protect silo from the damage. This problem is crucial in the case of silos with funnel flow.

In literature numerous papers can be found describing different measurement techniques as well as numerical simulations dedicated to flow processes analysis [11-13]. Both approaches, based on experiment and computing calculations, provide significant results, enriching expert knowledge about flow dynamics. In order to conduct a complex flow analysis, high precision and accurate measurement systems are necessary. Many systems provide possibilities of flow monitoring using images [14]. The sets of 2D or 3D images of granular distribution during flow allow to conduct very deeply analysis of process behaviour in a spatio-temporal sense [15]. For such investigations process tomography systems provide valuable information about process, especially during in-situ experiments $[18,19]$. X-ray tomography, one of the non-destructive techniques provide $3 \mathrm{D}$ information of the interior of the media, stands out as a unique tool to characterize processes that involve concentration changes, as in the case of gravitational flow [17-19].

The knowledge of density distribution within the bulk solid is of a major importance to understand and describe the flow behaviour of granular materials in silos while evaluating silo wall pressures, and to determine the flow type [6, 12, 20-22]. A comprehensive insight upon flow phenomena allows to develop more robust control system, save energy and achieve full quality product. This kind of collaboration between laboratory and industrial environment is based on rules, that wider knowledge about process can be used in control unit during real-time measurement data processing and improve the process.

The presented methodology aims at better understanding the flowing process of granular material in confined structure, thanks to use of X-ray imaging, development of dedicated image processing and analysis methods.

\section{X-ray laboratory setup}

In order to conduct a complex flow analysis, high precision and accurate measurement systems are necessary. Many systems provide possibilities of flow monitoring using images. The sets of $2 \mathrm{D}$ or $3 \mathrm{D}$ images of granular distribution during flow allow to conduct very deeply analysis of process behaviour in a spatio-temporal sense. For such an investigation process tomography systems provide valuable information about process, especially during in-situ experiments [19]. 
Visualizations of granular concentration distribution, based on the measurement of Xray attenuation of sample located between X-ray source and detector, are very useful for flow diagnosis (fig. 1), [19]. The X-ray measurement can be presented in form of 2D radiography or $3 \mathrm{D}$ tomography images. In term of $2 \mathrm{D}$ radiography image, the integration of the X-ray attenuation is directly connected to the granular concentration located on the linear path between the X-ray source and pixel localization on detector. During the experiments granular material showcases different properties, rice, sorghum and glass particles.
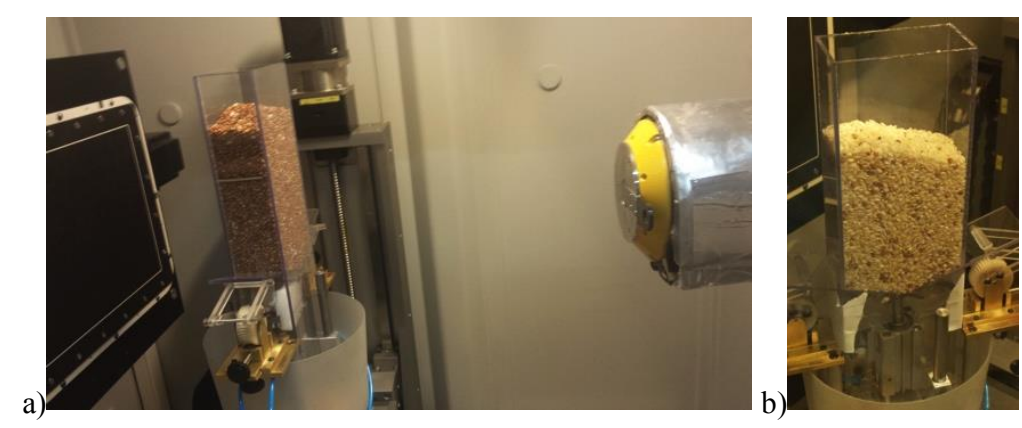

Fig. 1. Experimental set-up a) picture of silo model in X-ray tomography hutch, b) silo model with rice and sorghum mixture.

In order to carry out in situ granular flow experiments, an especially designed model silo with rectangular bin was used. The bin part was $10 \mathrm{~cm}$ wide, $5 \mathrm{~cm}$ deep and $20 \mathrm{~cm}$ high. The left and right hopper angles can be independently set to generate different types of flows, i.e. mass and funnel flows, with concentric/eccentric discharging modes (see fig1b).

The silo material is polycarbonate with $5 \mathrm{~mm}$ thick. The outlet width is manually set, what allows to control the discharge velocity. The time-lapse studies were performed at INSA- Lyon (France) using a GE Phoenix v|tome|x device (see fig. 1). The device is equipped with a high- energy X-ray microfocus source (up to $160 \mathrm{kV}$ ) with a $4 \mu \mathrm{m}$ spot size. The detector is equipped with a 1500x1920 pixels (with pixel size of $127 \mathrm{X} 127 \mu \mathrm{m} 2$ ), which allows to scan objects of height $20 \mathrm{~cm}$. During measurement the silo model was not rotated, as for $3 \mathrm{D}$ imaging, so position of silo was the same during all experiments. In figure 2 is presented example of raw radiography image.

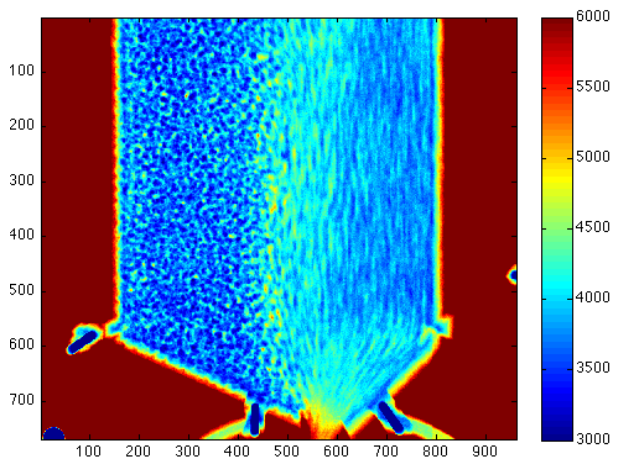

Fig. 2. Radiography image for eccentric flow - left hopper angle $20^{\circ}$ and right $40^{\circ}$.

Before calculating the Gray-Level Co-Occurrence Matrix (GLCM) [23-25], the normalization procedure for raw radiography image was applied in order to improve the 
image contrast [19]. Such approach expedites the distinction between the stagnant and flowing zones inside granular material. However the most difficult problem is to segment the flowing zone into at least 2 different regions. Three different zones, creating characteristic spatial patterns, are visible in the presented image (fig. 2). For such type of images, texture analyzed can be applied to determine the silo area characterized by individual spatial distribution of gray pixels values distributions.

\section{GLCM matrix analysis}

The spatial relationship, texture analysis, of pixels in radiography images can be examined by applied the gray-level co-occurrence matrix (GLCM). The characterization of the texture of an image is conducted by calculating how often pairs of pixel in a specified spatial relationship occur in an image. This information is collected in GLCM matrix and after can be extracted by used statistical measures of this matrix [25].

In the first step of presented method the GLCM matrix is calculated and after its features are determined, which allow to analyze the texture patterns in images. The statistical parameters of GLCM are extracted based on the normalized, symmetric GLCM matrix. The matrix values calculation can be described as:

$$
\boldsymbol{G L C M}_{\Delta i, \Delta j}(v, w)=\sum_{v, w=1}^{K}\left\{\begin{array}{c}
1, \text { if } I(i, j)=v \text { and } I(i+\Delta i, j+\Delta j)=w \\
0, \text { otherwise }
\end{array}\right.
$$

where:

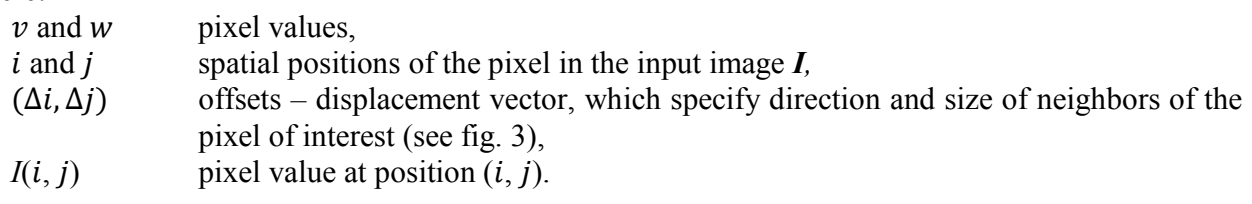

In the presented equation the size of GLCM matrix is equal to $K \mathrm{x} K$, where $K$ indicates number of grey levels in input image, so before calculation of GLCM matrix the grey level should decreased to reasonable value.

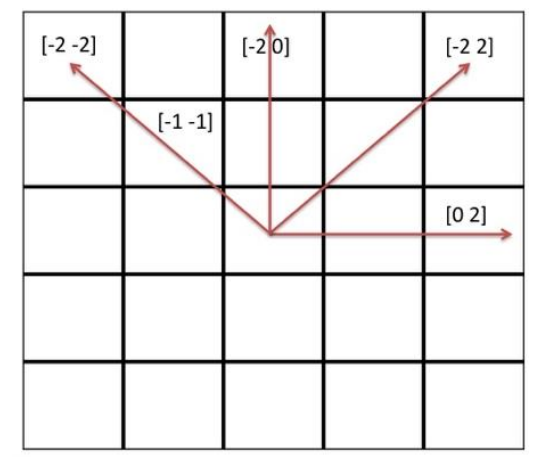

Fig. 3. Definition of size and orientation of neighbourhood for GLCM matrix calculation, example for displacement vector $(2,2)$.

The various features, which measure the gray-level distribution, can be calculated based on GLCM matrix values. In presented paper were chosen homogeneity and entropy:

$$
\text { homogeneity }=\sum_{v, w=1}^{K} \frac{1}{1+|v-w|} p(v, w)
$$




$$
\text { entropy }=-\sum_{v, w=1}^{K} p(v, w)^{2} * \log (p(v, w))
$$

where:

$$
\begin{array}{ll}
p(v, w) & \text { depicts GLCM's values as probabilities after normalization, } \\
\sigma_{x}, \sigma_{y}, \mu_{x}, \mu_{y} & \begin{array}{l}
\text { standard deviations and means of } p_{x}(v)=\sum_{j=1}^{K} p(v, w) \text { and } p_{y}(j)=\sum_{i=1}^{K} p(v, w) \\
\text { respectively. }
\end{array}
\end{array}
$$

All features were calculated locally, for predefined displacement vector and number of grey levels $K$. The presented results were obtained for number of discretized grey level $K=8$ (in input radiography image) and for offsets [-1 0] and [1 0$]$ (fig. 3). The size of neighbourhood for calculating local GLCM was $15 \times 15$. The calculations were conducted in Matlab ${ }^{\circledR}$ numerical computing environment.

\section{Results and discussion}

The radiography images were preprocessed before calculation of GLCM matrix. The applied normalization procedure of radiography image was described in [19]. The normalized radiography image allows to visualize the distribution of bulk solid concentration in comparison to initial state of silo discharging process (before open the silo outlet). Additionally the procedure eliminates influence of the silo wall on measurement results. In figure 4 are presented two radiography images, before normalization (fig. 4a), and after normalization (fig. $4 \mathrm{~b}$ ). The normalization calibrates data into range $<0,1>$. The value 0 means empty space, radiography was obtained for empty silo and value 1 means values of material concentration obtained at initial stage of silo discharging process, radiography was obtained for full silo. The presented results ware taken for initially dense packing and for eccentric flow.
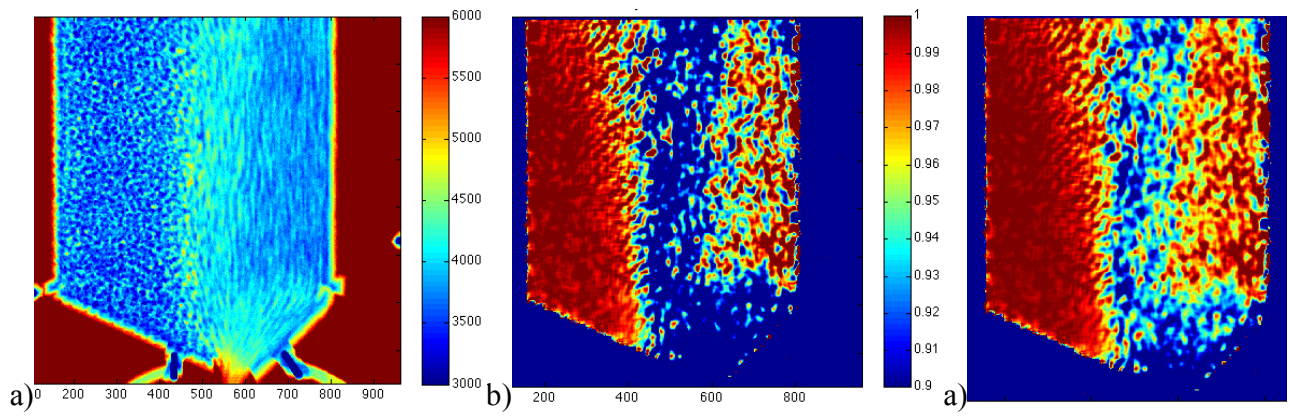

Fig. 4. Radiography images, a) scaled raw data, b) normalized data, c) filtering of normalized data.

The second step in pre-processing procedure was applied image filtering (average filter with size $7 \times 7$ ). In figure $4 \mathrm{c}$ is presented the example of normalized radiography image after filtering. The averaging filter allowed to visualize the granular material distribution in easier way to determine homogeneities of material structures. The examples presented in Figure 5 were obtained for middle and final step of silo discharging process. The homogeneity images (see fig. 5b) can be interpreted as level of similarity in granular material distribution; a higher value depicts more homogeneous area of pixels values, what is important for better interpretation of images. Entropy images provide similar information, however the scale values are much different than for homogeneity images.

The analysis of the obtained results allows to state, that it is possible to divide a silo space into part, characterized by similar flow pattern. Analysing of the homogenous image presented for other time point (fig. 5b) shows that high level of homogeneous is presented in two segments of silo (left and right part). It is also possible to distinguish, from the rest 
of silo, main funnel flow area with lower homogeneity value $(\sim 0.925)$. The combine of the normalized radiography image (fig. 6b) and GLCM homogenous image (fig. 6a) should allow to easier distinguish 3 zones of flow. In the future work this approach will be developed as well applied other GLCM features to prepared set of data for classification procedure using k-means algorithm.
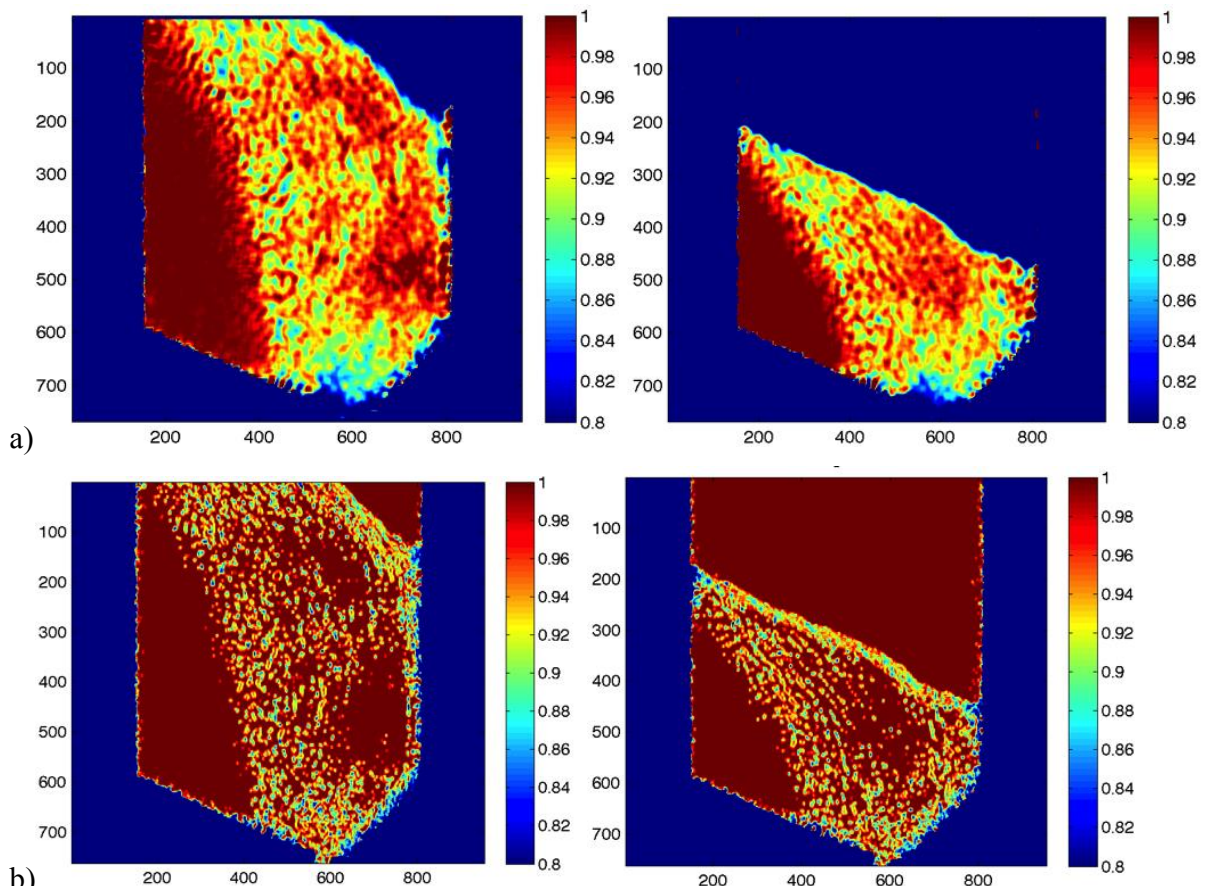

b)
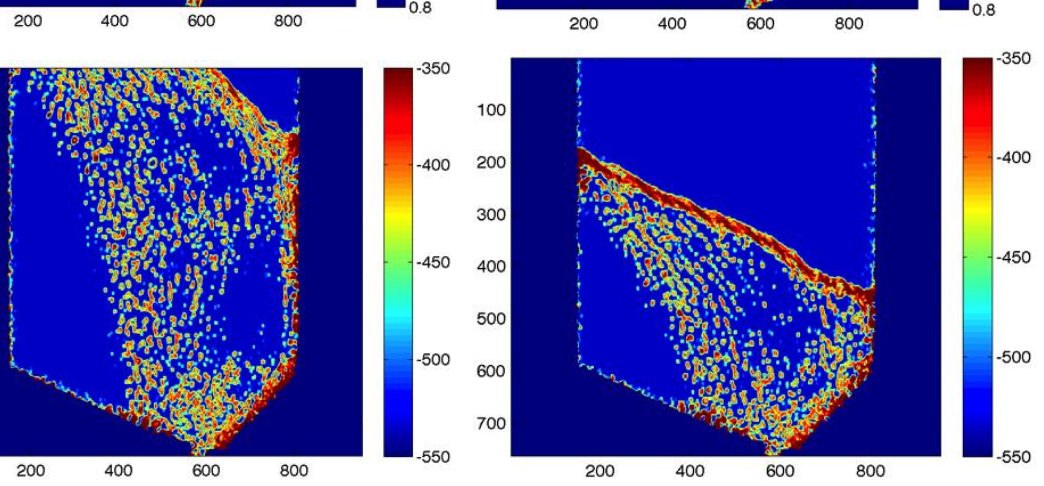

c)

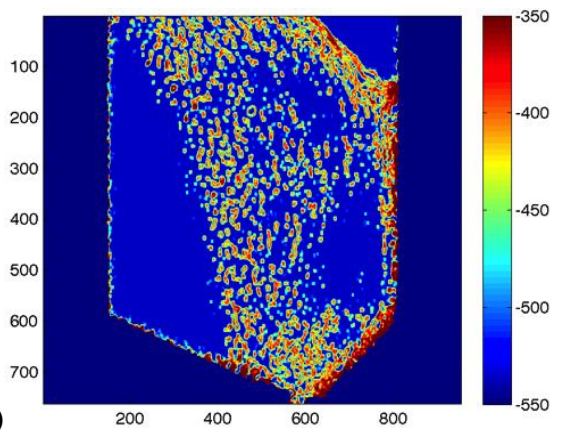

Fig. 5. Images of GLCM statistical features, a) normalized radiography after filtering, b) homogeneity, c) entropy. 

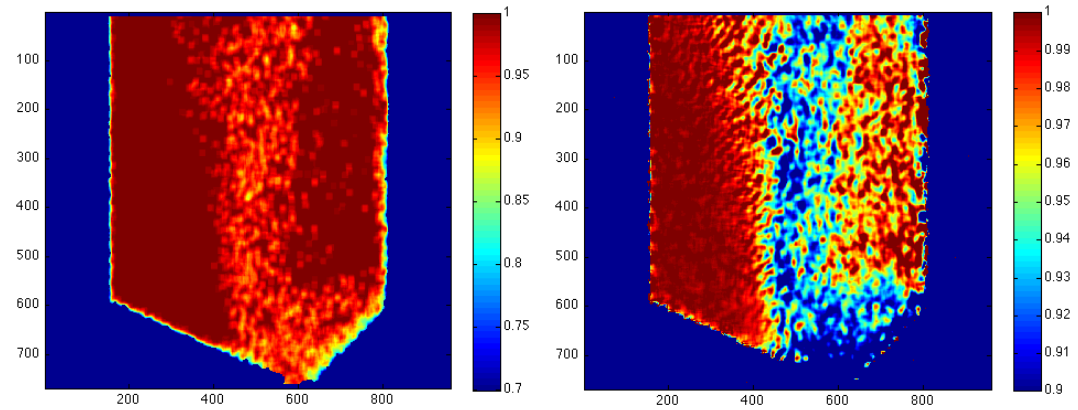

Fig. 6. Data after averaging filtering $(7 \times 7)$, a) the normalized radiography image, b) image of homogeneity.

\section{Conclusions}

The paper presents preliminary results of developing computer system for analysing of silo discharging process. The aim of instigation is a prepared image processing algorithm which allows to divide the silo area into homogenous zones in context of similarity in distribution of bulk solid concentration. The knowledge about the level of concentration changes during silo discharging in separate zones provides new point of view for investigated gravitational flow. Silo flow segmentation into homogeneous structures, in terms of granular material concentration changes, will improve understanding of flow phenomena for concentric and eccentric flow with various properties granular particles.

Similarity in pattern of material distribution occurring in radiography images can be analysed with the aid of texture analysis methods. The proposed concept bases on analysis of GLCM matrix features. The obtained GLCM features show that is possible to distinguish silo parts, much easier than for raw or even normalized radiography image. However in order to achieve the expected results necessary is to conduct more sophisticated analysis of GLCM matrix. Especially, the combination of different features and radiography images should provide vector of data, which can be used for conducting the image segmentation.

This work is supported by the National Science Centre, Poland (NCN OPUS10 2015/19/B/ST8/02773)

\section{References}

1. D. Schulze, Powders and Bulk Solids (Springer, 2008)

2. J.P.K.Seville, U. Tuzun, R. Clift, Processing of Particulate Solids, (Blackie Academic, London, 1997)

3. C. J. Brown, J. Nielsen, Silos: fundamentals of theory, behavior, and design, (E \& FN Spon, 1998)

4. Buick J.M., J. Chavez-Sagarnaga, Z. Zhing, J.Y. Ooi, D.M. Pankaj, D.M. Cambell, C.A. Greated, Journal of Engineering Mechanics ASCE, 131, 299 (2005)

5. K. Wilde, J. Tejchman, M. Rucka, N. Niedostatkiewicz, Powder Technology, 198, 38 (2010)

6. M. Wojcik, J. Tejchman, Powder Technology, 192, 298 (2009)

7. V. Mosorov, Flow Pattern Tracing for Mass Flow Rate Measurement in Pneumatic Conveying Using Twin Plane Electrical Capacitance Tomography, Particle \& Particle Systems Characterization, 25, 259, (2008) 
8. A. Romanowski, K. Grudzien, R.G. Aykroyd, R.A. Williams, Part. Part. Syst. Charact., 23, 289 (2006)

9. M.L. Dhoriyani, K.K. Jonnalagadda, R.K. Kandikatla, K.K. Rao, Powder Technology, 167, 55 (2006)

10. R. Maiti, G. Das, P. K. Das, Powder Technology, 301, 1054 (2016)

11. R. Balevičius, R. Kačianauskas, Z. Mróz, Irena Sielamowicz, Powder Technology, 187, 159 (2008)

12. J. Tejchman J., Confined Granular Flow in Silos, Experimental and Numerical Investigations (Springer, 2013)

13. T. Rymarczyk, P. Tchórzewski, P. Adamkiewicz, K. Duda, J. Szumowski, J. Sikora, IEEE Sensors Journal, 17, 8166 (2017)

14. J. Kryszyn, D. M. Wanta and W. T. Smolik, IEEE Sensors Journal, 17, 8107 (2017)

15. R. Banasiak, R. Wajman, T. Jaworski, P. Fiderek, H. Fidos, J. Nowakowski, Dominik Sankowski, International Journal of Multiphase Flow, 58, 1 (2014)

16. M. Niedostatkiewicz, J. Tejchman, K. Grudzień, Z. Chaniecki, Chemical Engineering Research and Design, 88, 1037, (2010)

17. R.L. Michalowski, Powder Technology, 39, 29 (1984)

18. C. Chen, P. W. Woźniak, A. Romanowski, M. Obaid, T. Jaworski, J. Kucharski, K. Grudzień, S. Zhao, and M. Fjeld, ACM Trans. Intell. Syst. Technol., 7, 25, (2016)

19. K. Grudzień K., M. Niedostatkiewicz, J. Adrien, E. Maire, and L. Babout, Powder Technology, 224, 196, (2012)

20. H. Ahn, Z. Başaranoğlu, M. Yılmaz, A. Buğutekin, M. Zafer Gül, Powder Technology, 186, 65 (2008)

21. C. González-Montellano, Á. Ramírez, E. Gallego, F. Ayuga, Chemical Engineering Science, 66, 5116 (2011)

22. C. González-Montellano, F. Ayuga, J. Y. Ooi, Granular Matter, 13, 149 (2011)

23. R. Shenbagavalli, K. Ramar, Bonfring International Journal of Advances in Image Processing, 1, 15, (2011)

24. R. M. Haralick, K. Shanmugam, I. Dinstein, IEEE Transactions on Systems, Man, and Cybernetics, 3, 610, (1973)

25. Q. Chen, E. Agu, International Conference on Healthcare Informatics, 453 (Dallas, TX, 2015) 